OPEN ACCESS

Edited by:

Santosh Kumar,

Liaocheng University, China

Reviewed by:

Sushank Chaudhary,

Quanzhou Institute of Equipment manufacturing, Chinese academy of

Sciences, China, China

Dr. Shyam Akashe,

ITM University, India

*Correspondence:

Amit Grover

amitgrover321@gmail.com

Specialty section:

This article was submitted to

Optics and Photonics,

a section of the journal

Frontiers in Physics

Received: 18 July 2021

Accepted: 27 July 2021

Published: 31 August 2021

Citation:

Singh M, Pottoo SN, Suvidhi, Dewra S,

Rishabh, Grover A, Manikandan A and

Sheetal A (2021) Performance

Investigation of a High Data Rate Mode

Division Multiplexed-Free Space

Optics Link Under Harsh

Weather Conditions.

Front. Phys. 9:743545.

doi: 10.3389/fphy.2021.743545

\section{Performance Investigation of a High Data Rate Mode Division Multiplexed-Free Space Optics Link Under Harsh Weather Conditions}

\author{
Mehtab Singh ${ }^{1,2}$, Sahil Nazir Pottoo ${ }^{3}$, Suvidhi ${ }^{4}$, Sanjeev Dewra ${ }^{5}$, Rishabh ${ }^{6}$, Amit Grover $^{5 *}$, \\ A. Manikandan ${ }^{7}$ and Anu Sheetal ${ }^{8}$ \\ ${ }^{1}$ Department of Engineering and Technology, Guru Nanak Dev University, Jalandhar, India, ${ }^{2}$ Department of Electronics and \\ Communication Engineering, SIET, Amritsar, India, ${ }^{3} \mathrm{Center}$ for Interdisciplinary Programs, Indian Institute of Technology \\ Hyderabad, Telangana, India, ${ }^{4}$ Department of Computer Science and Engineering, Punjab Engineering College (Deemed to be \\ University), Chandigarh, India, ${ }^{5}$ Department of Electronics and Communication Engineering, Shaheed Bhagat Singh State \\ University, Ferozepur, India, ${ }^{6}$ Department of Computer Application, ABES Engineering College, Ghaziabad, India, ${ }^{7}$ Department of \\ Electronics and Communication Engineering, Vivekanandha College of Technology for Women, Tiruchengode, India, \\ ${ }^{8}$ Department of Engineering and Technology, Guru Nanak Dev University, Gurdaspur, India
}

The requirement of high data rate information transmission is rising exponentially for supporting different services including social networking, web streaming, and biomedical sensor data transmission. Such services required high channel bandwidth with secure information transmission and immunity to electromagnetic interference. Radio over free space optics (RoFSO) is witnessed as a promising technological solution to provide high data rate transmission over free space channel. We report on the design of a $2 \times 10 \mathrm{~Gb} / \mathrm{s}-$ $10 \mathrm{GHz}$ RoFSO transmission system using the mode division multiplexing technique and evaluate its transmission performance over varying levels of dust weather conditions. The comparison of non-return to zero (NRZ) and return to zero (RZ) binary digital optical modulation techniques is carried out in the proposed system. It is found that the proposed system using NRZ modulation serves $14.5 \mathrm{~km}$ transmission range; however, in the case of $\mathrm{RZ}$ modulation, it was restricted to $10 \mathrm{~km}$ for a target bit error rate (BER) of $10^{-6}$, thus resulting in a noticeable link enhancement of $4.5 \mathrm{~km}$. Also, we demonstrate NRZ-based MDM-RoFSO link performance and availability in dust weather conditions using the BER, maximum reachable link range, and eye diagram as key performance parameters. We obtain a reliable transmission of $20 \mathrm{~Gb} / \mathrm{s}-20 \mathrm{GHz}$ data through $\mathrm{HGOO}$ and $\mathrm{HGO} 1$ channels over a link range of 2500-108 m depending on the external dust weather condition. Furthermore, since this investigation shows the feasibility of RoFSO for small size cells, which is an essential feature of $5 \mathrm{G}$ mobile network, the proposed system can thus be implemented as a backhaul/fronthaul link for high-band (above $6 \mathrm{GHz}$ ) $5 \mathrm{G}$ services and for providing secure transmission of biomedical sensor data.

Keywords: HG, biomedical sensor data, MDM, RoFSO, 5G technology 


\section{INTRODUCTION}

In the present-day telecommunications sector, the base transceiver station (BTS)-BTS connection is established through fiber optic trunk cables in which voice/data are transmitted at high speed using radio over fiber (RoF) technology. Also, in order to establish a BTS-user communication link, a radio-frequency (RF) network is deployed. Nevertheless, fiber installation constrictions both in urban and rural areas, excessive infrastructure costs, and hefty spectrum pricing in the case of RF technology make such approaches unattractive and unfavorable for the establishment of next-generation wireless networks. To overcome this trouble, a radio over free space optics (RoFSO) link, where the RF signal will be transmitted between BTSBTS or BTS-user using an optical carrier (up-conversion) and air/free space as the medium, is a viable solution. RoFSO technology provides unlicensed spectrum operation, interference, and interception-free transmission within a short deployment period and little expense [1]. To enhance the spectral efficiency and information-carrying capacity of RoFSO links, the mode division multiplexing (MDM) technique is incorporated in which independent RF signals are transmitted over distinct spatial modes of a single frequency laser beam [2-6]. Recently, Satea et al. proposed that FSO systems employing multiple channels with erbium-doped fiber amplifiers (EDFAs) work efficiently under high attenuation environments such as heavy fog and dust [7]. Alaa et al. analyzed the FSO system performance in fog and sandstorm conditions by changing the operating wavelength, receiver diameter aperture, photodetector type, and the modulation technique. They found that both the dense fog and dust triggered 87 and $95 \%$ power loss, respectively, at a transmission range of $1 \mathrm{~km}$ [8]. Maged et al. put forth an experimental evaluation of an all-optical hybrid FSO/RF link carrying $5 \mathrm{G}$ signals in a dust channel. They reported that the RF link with low bandwidth works well in dense dust and the FSO operates once the weather improves beyond a definite threshold. As a consequence, the FSO/RF parallel link can be a first-rate choice towards overcoming dust effects [9]. Matthew et al. proposed a novel visibility and dust absorption model after using visibility and absorption measurements made farther from the source $(10-100 \mathrm{~km})$ to demonstrate the impact of reduced dust particle size over the regional distance from the source [10]. Haichao et al. described that in addition to wavelength, particle sizes of sand and dust affect the laser beam attenuation [11]. Zabih et al. reported that the FSO link performance can be improved by increasing the launched optical power and selecting adequate line speed under sandstorm conditions [12]. The current research in RoFSO is mainly focused on fog- and smoke-induced attenuation [13-16]. Intensive investigation and modeling of the dust effects on the RoFSO channel are yet to grab the attention of researchers. Very limited or basically no attempt has been made in this course, and the development and investigation of MDM-based RoFSO transmission for dust environment has not been reported till date. In arid and semiarid regions, the effect of dust will remain paramount for any upcoming installation of the RoFSO network in 5G and smart city applications [17]. It is straightforward that the propagation prediction under dust storms regarding signal attenuation, maximum reachable distance, and error performance will be the solemn challenge for the setting up of the RoFSO communication network.

This work reports the designing and performance evaluation of an MDM-RoFSO transmission for varying levels of dust and clear climate conditions. This research article is structured as follows: FSO Evaluation in Dust Channel discusses the numerical model for dust storm characteristics and attenuation in FSO. Simulation Setup Description describes the proposed simulation

TABLE 1 | Dust storm classification established on a visibility basis.

\begin{tabular}{lccc}
\hline Type of dust & Severe dust storm & Dust storm & Blowing dust \\
\hline Depiction & Dense & Moderate & Dight \\
Visibility, $\boldsymbol{V}(\mathbf{k m})$ & $<0.2$ & $0.2-1$ & $1-10 \quad$ Very light
\end{tabular}

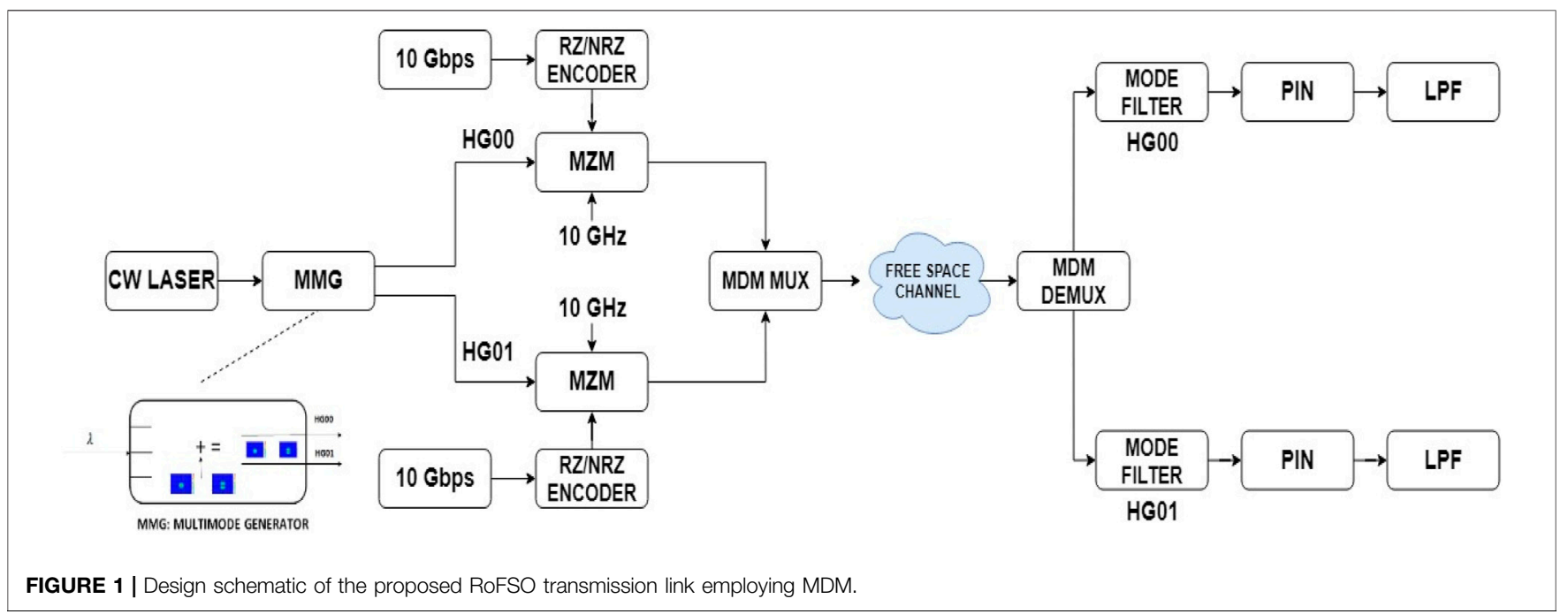



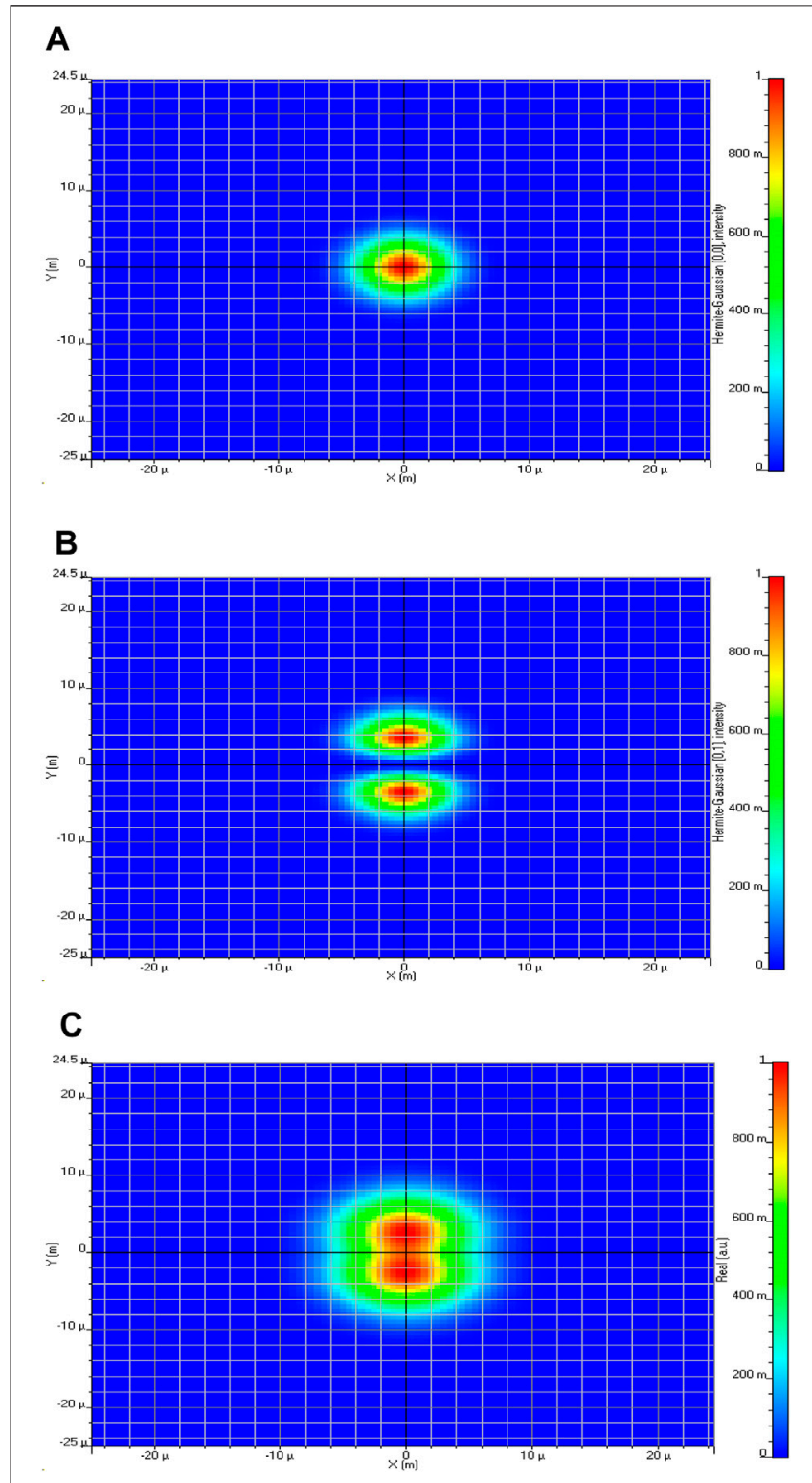

FIGURE 2 | Spatial intensity pattern of the (A) HG00 mode, (B) HG01 mode, and (C) transmitted MDM signal.

setup and parameters. Results and Discussion presents the simulation results, and Conclusion concludes the paper.

\section{FSO EVALUATION IN DUST CHANNEL}

In FSO, the chosen signal wavelength belongs to the atmospheric transmission window which provides low molecular absorption since absorption cross-section $\sigma_{a}$ is negligible, that is, $\sigma_{a} \sim 0$ [18]. Therefore, the contribution of absorption to the total attenuation coefficient is meager in comparison to the scattering effect [19]. Different environmental factors for instance fog, rain, storm, snow, smoke, and dust causes scattering of optical beam [20, 21]. To
TABLE 2 | Simulation parameters considered for the proposed link design $[30,31]$.

\begin{tabular}{lc} 
Parameter & Value \\
\hline Operating frequency & $193.1 \mathrm{THz}$ \\
Input power & $14 \mathrm{dBm}$ \\
Laser linewidth & $100 \mathrm{e}-012 \mathrm{MHz}$ \\
Bit rate & $10 \mathrm{Gbps} / \mathrm{channel}$ \\
Transmitter aperture diameter & $10 \mathrm{~cm}$ \\
Receiver aperture diameter & $20 \mathrm{~cm}$ \\
Beam divergence angle & $0.25 \mathrm{mrad}$ \\
PIN responsivity & $1 \mathrm{AW}$ \\
LPF cutoff frequency & $0.75 \times \mathrm{bit} \mathrm{rate}$ \\
Sequence length & 1,024 \\
Sample per bit & 32 \\
\hline
\end{tabular}
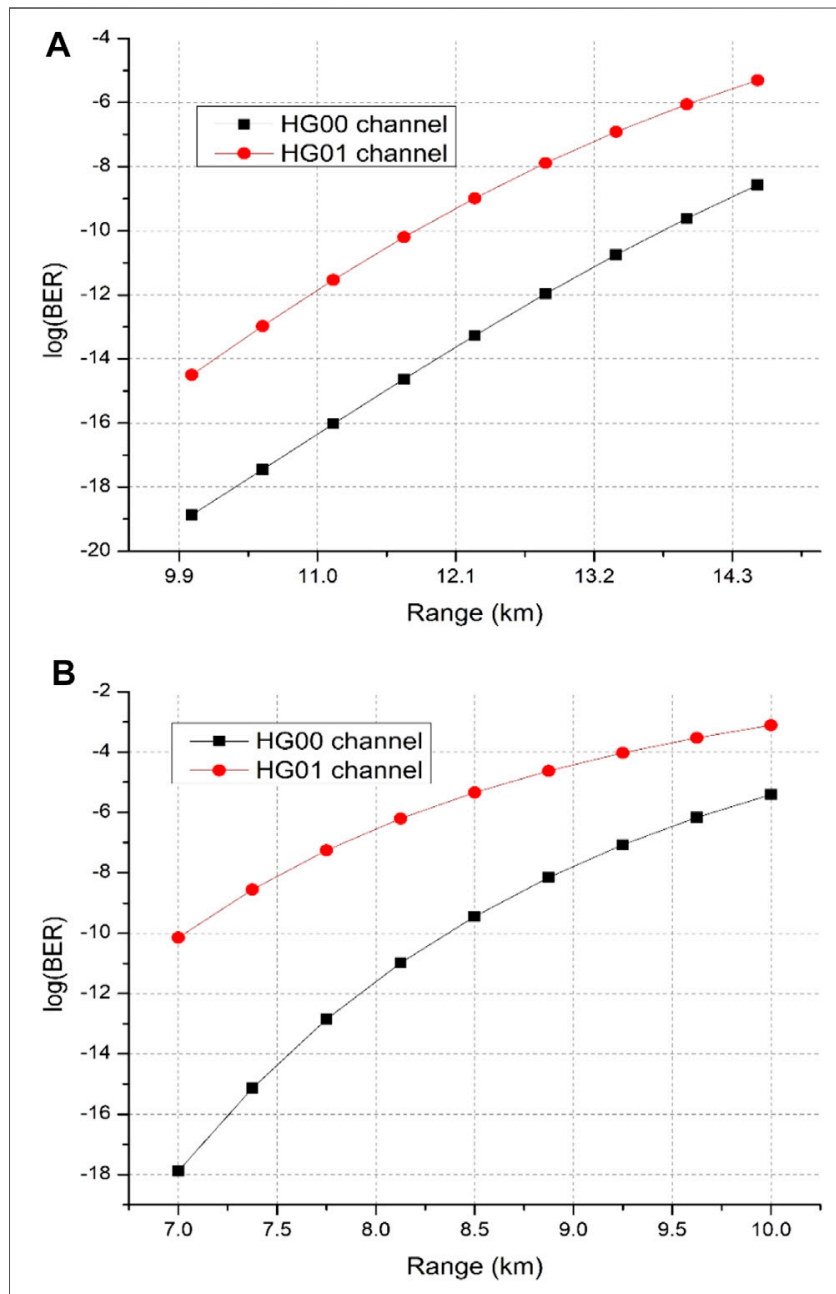

FIGURE 3 | log (BER) V/s transmission distance for clear weather using (A) NRZ modulation and (B) RZ modulation.

investigate the consequences of these conditions on the FSO, one of two existing approaches can be employed. The first approach utilizes theoretic-based theorems such as Mie theory. Nevertheless, this approach involves some parameters that may not be accessible at the installation site, for example, refractive index, particle size, and distribution. [22]. 

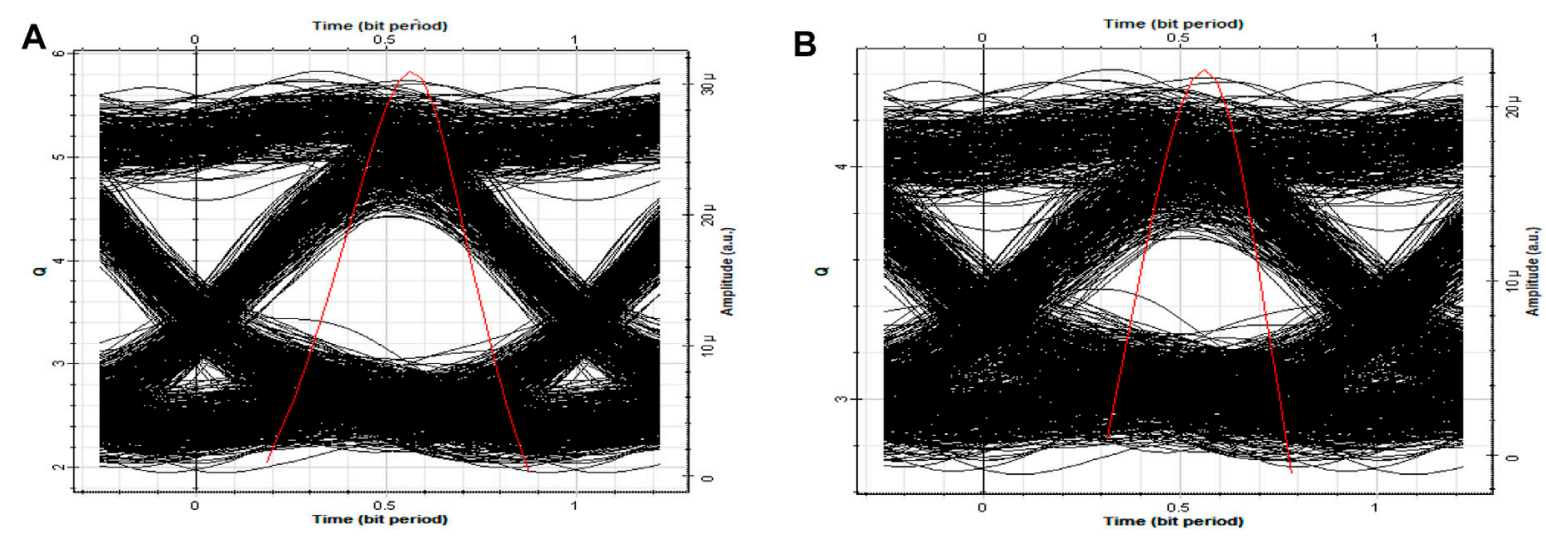

FIGURE 4 | Eye diagram using the proposed NRZ-based MDM-RoFSO link at $14.5 \mathrm{~km}$ transmission distance under clear weather for the (A) HG00 channel and (B) HG01 channel.

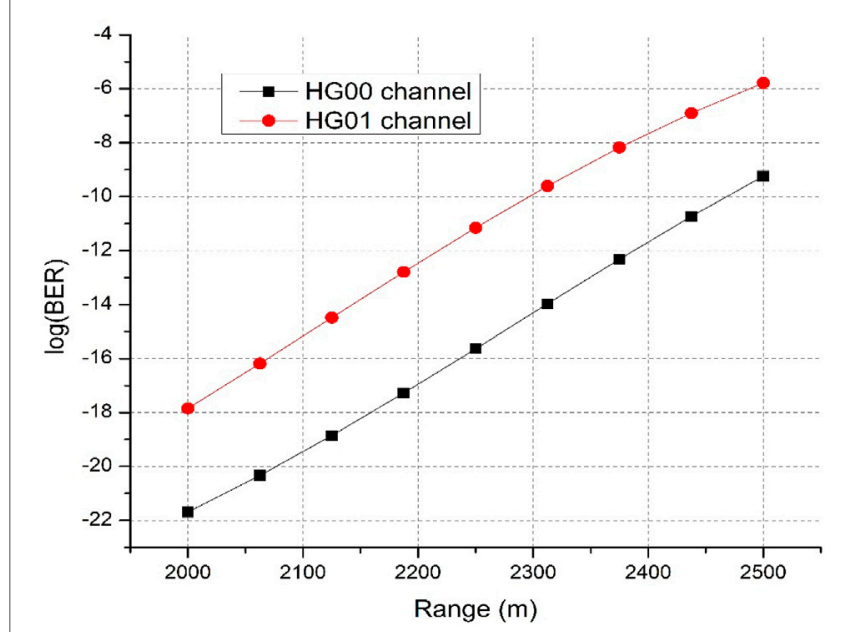

FIGURE 5 | log (BER) v/s transmission distance for very light dust. $(V \leq 10 \mathrm{~km})$.

The second approach used in the literature for the calculation of attenuation in FSO for real-world applications rests on experimental remark [22]. This technique is based on experiential models established by means of visibility range statistics to identify the propagation path characteristics [23]. Link visibility information can be acquired from meteorological stations situated near the setup area. In [24], visual range is described as the distance to an entity at which the picture distinction falls to $2 \%$ of the original ocular contrast (100\%) along the transmission distance usually referred to as the Koschmieder law. This $2 \%$ falloff value recognized as the visual threshold $T_{v i s}$ is adopted here so as to follow the Koschmieder law as opposed to the $T_{v i s}=5 \%$ considered in aviation operations $[25,26]$. The visibility is computed at $550 \mathrm{~nm}$ wavelength since the highest solar radiation concentration occurs here. Visibility indicates the degree of sternness of the dust gale. Small visibility specifies the elevated concentration of dust elements in the free space, and vice versa.

Depending on the visibility, dust storms are classified into four groups as specified in Table 1. Type 1 is dust haze (very light dust) wherein widespread dust particles drift up and happen to remain suspended evenly in the atmosphere as a result of dust storm that begins at a large distance from the monitoring point. The visibility associated with this occasion is about $10 \mathrm{~km}$ or less. Type 2 is blowing dust (light dust) in which the dust is blown in the direction of monitoring position by the action of winds. As a result, the visibility drops between 1 and $10 \mathrm{~km}$. Type 3 is dust storm (moderate dust) where strong winds bluster additional dust elements inside the observation area and the visibility decreases between 0.2 and $1 \mathrm{~km}$. Finally, type 4 is severe dust storm (dense dust) which arises after a large volume of dust particles are blown by violent winds, and the visibility further drops below $0.2 \mathrm{~km}$. The visibility $V(\mathrm{~km})$ in terms of $\alpha$ and $T_{v i s}$ takes the form [22]:

$$
V=-\frac{10 \log T_{v i s}}{\alpha}
$$

where $\alpha$ is the specific atmospheric attenuation coefficient expressed in $(\mathrm{dB} / \mathrm{km})$ and is determined from the light wave transmittance $T$ at $550 \mathrm{~nm}$ and transmission distance $d(\mathrm{~km})$ using the Beer-Lambert law as [27]:

$$
\alpha=\frac{10 \log T}{4.343 d} \text {. }
$$

Moreover in [28], the FSO signal attenuation model as a function of the visibility for desert environment that is prone to frequent dust storms is obtained as:

$$
\alpha=52 \times V^{-1.05}
$$

Eq. 3 holds under the condition that the operating wavelength used in FSO communication is $1,550 \mathrm{~nm}$ since it provides lowest atmospheric attenuation and minor absorption loss, besides its technology is mature in terms of fabrication of optoelectronic devices.

\section{SIMULATION SETUP DESCRIPTION}

The proposed MDM-based RoFSO link setup employing RZ and NRZ binary digital optical modulation is illustrated in Figure 1, 

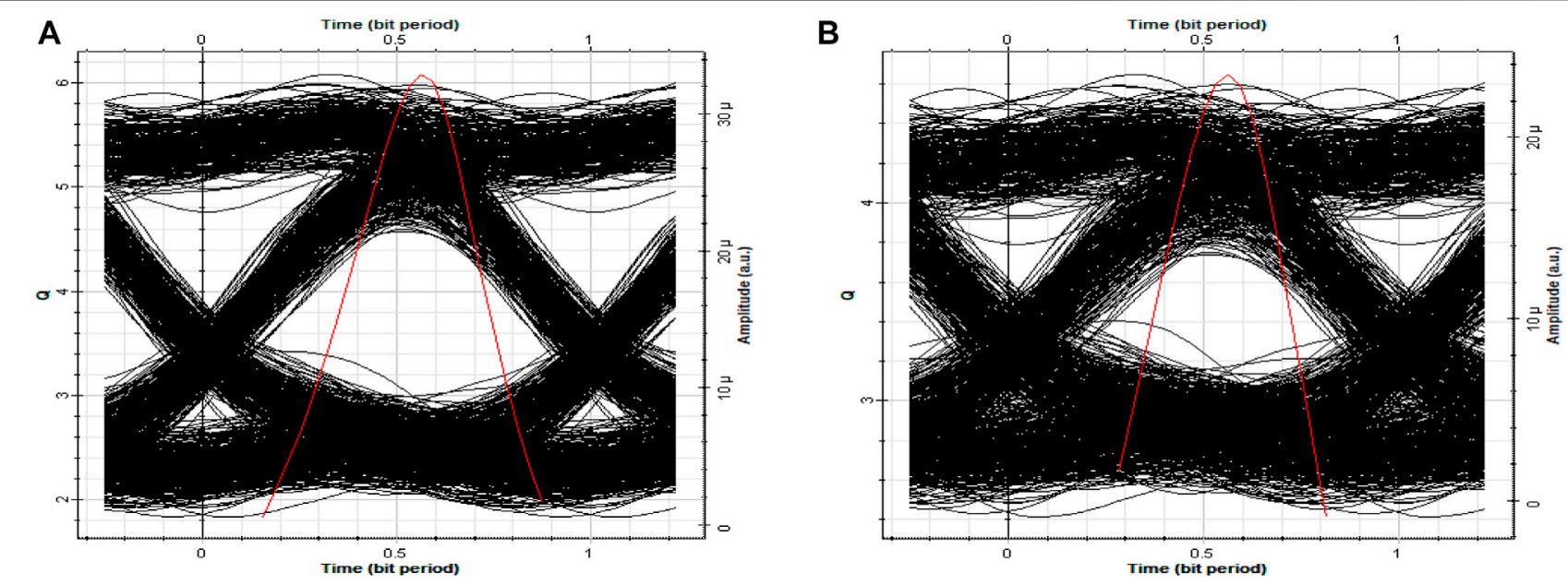

FIGURE 6 | Eye diagram using the proposed NRZ-based MDM-RoFSO system at 2,500 m transmission distance under very light dust for the (A) HGO0 channel and (B) HG01 channel.

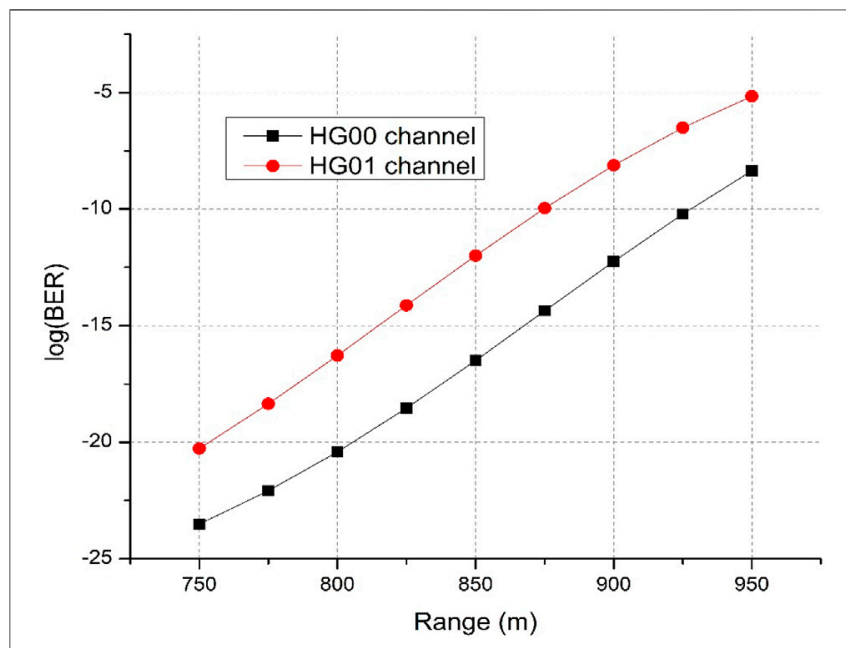

FIGURE 7 | log (BER) v/s transmission distance for light dust.

which has been designed using the Optisystem ${ }^{\mathrm{TM}}$ photonic software. Two independent $10 \mathrm{GHz}$ RF signals are modulated over different HG mode beams, that is, HG00 and HG01 and transported over the FSO channel. Eq. 4 mathematically describes the intensity profiles of HG modes (Figures 2A,B) as [29]:

$$
\begin{aligned}
\psi_{p, q}(x, y) & =H_{p}\left[\left(\frac{\sqrt{2} x}{w_{o x}}\right) \exp \left(-\frac{x^{2}}{w_{o x}^{2}}\right) \exp \left(j \frac{\pi x^{2}}{\lambda R_{o x}}\right)\right] \\
& \times H_{q}\left[\left(\frac{\sqrt{2} y}{w_{o y}}\right) \exp \left(-\frac{y^{2}}{w_{o y}^{2}}\right) \exp \left(j \frac{\pi y^{2}}{\lambda R_{o y}}\right)\right],
\end{aligned}
$$

where $p$ and $q$ denote mode dependencies on the $x$ and $y$ axes, $\mathrm{R}$ signifies the radius of curvature, $w_{o}$ indicates the spot size, and $H_{p}$ and $H_{q}$ represent Hermite polynomials. In our proposed design, a continuous wave (CW) laser at 193.1 THz together with an MMG is used to generate two different HG modes. Figure 2 depicts the spatial profile of $\mathrm{HG}$ modes. Figure $\mathbf{2 C}$ demonstrates the spatial profile of the $2 \times 10 \mathrm{~Gb} / \mathrm{s}-10 \mathrm{GHz}$ MDM-RoFSO transmitted signal.

A $10 \mathrm{~Gb} / \mathrm{s}$ information-carrying signal is produced by a pseudorandom bit sequence generator (PRBS) for distinct channels and encoded using RZ/NRZ schemes. In RZ-encoded signal, bit " 1 " is transported by an optical pulse with half bit period. Whereas in NRZ-encoded signal, bit "1" is transported through an optical signal of entire bit period, while in place of bit " 0 ", no optical signal is conveyed. A Mach-Zehnder modulator (MZM) is utilized to superimpose the encoded information onto a $10-\mathrm{GHz}$ RF signal and a distinct HG mode. This information signal is subsequently communicated into the FSO channel through HG00 and HG01 modes of the $193.1 \mathrm{THz}$ frequency channel.

Mathematically, the signal received after FSO transmission can be modeled as [32]:

$$
P_{r x}=P_{t x}\left[\frac{D_{r x}^{2}}{\left(D_{t x}+\theta d\right)^{2}}\right] 10^{-\alpha d / 10},
$$

where $P_{r x}$ and $P_{t x}$ denote the received and transmitted optical power, respectively, $D_{r x}$ and $D_{t x}$ denote the receiver and transmitter antenna diameter, and $\theta$ is the divergence angle. The system parameters considered in the proposed link design are listed in Table 2. The coefficient of atmospheric attenuation for clear sky is $0.14 \mathrm{~dB} / \mathrm{km}$ and $6.73 \mathrm{~dB} / \mathrm{km}$ for very light dust, 25.11 for light dust, $107.66 \mathrm{~dB} / \mathrm{km}$ for moderate dust, and $297.38 \mathrm{~dB} / \mathrm{km}$ for dense dust [33]. At the receiver end, separates modes are filtered using a mode filter [34, 35]. A spatial positive-intrinsic-negative (PIN) photodiode converts the received optical signal into its electrical equivalent which is followed by low-pass filtering to retrieve the original baseband signal. 

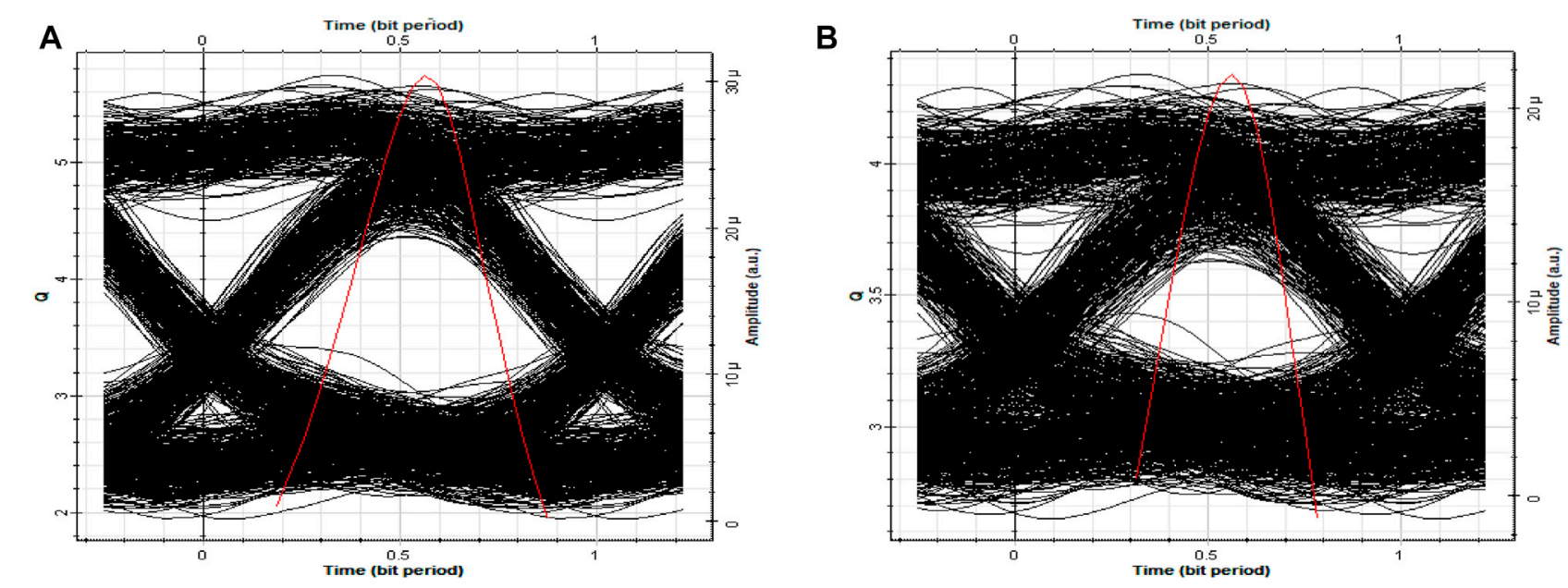

FIGURE 8 | Eye diagram using the proposed NRZ-based MDM-RoFSO link at $950 \mathrm{~m}$ transmission distance under light dust conditions for the (A) HG00 channel and (B) HG01 channel.

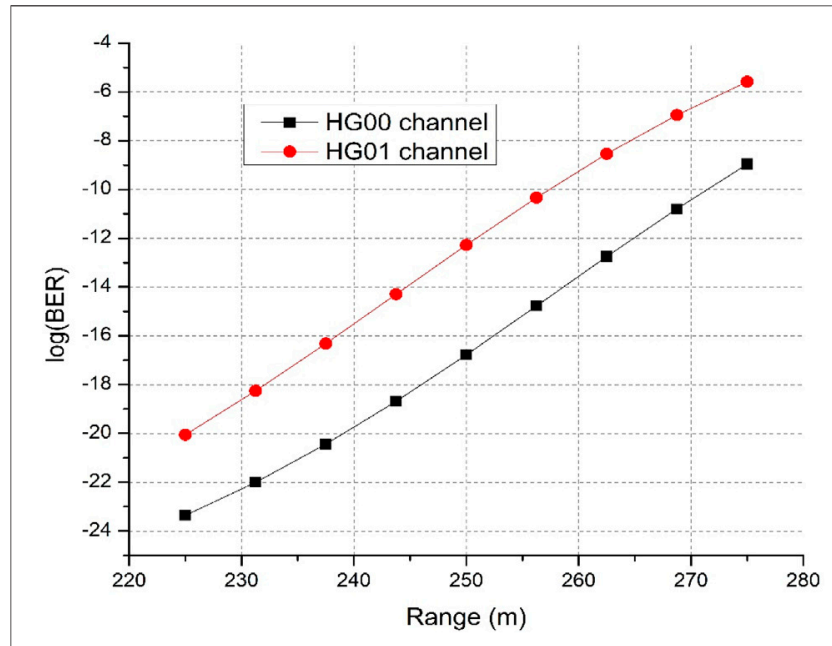

FIGURE 9 | log (BER) v/s transmission distance under moderate dust conditions.

\section{RESULTS AND DISCUSSION}

Firstly, we compare the performance of NRZ and RZ modulationbased MDM-RoFSO link under a clear climate. Secondly, we investigate the proposed MDM-RoFSO link utilizing NRZ modulation for four different dust storm conditions. The performance analysis has been carried out with regard to BER, transmission range, and eye diagram.

Figure 3 presents RZ/NRZ-MDM-RoFSO transmission system performance in terms of BER and range under clear weather conditions where each HG mode (HG00 and HG01) carries independent $10 \mathrm{~Gb} / \mathrm{s}-10 \mathrm{GHz}$ information. Though BER of the system degrades with increasing transmission distance for both the channels, it can be found from Figure 3A, using NRZ modulation scheme, the maximum achievable link range is
$14.5 \mathrm{~km}$ while it decreases to $10 \mathrm{~km}$ for $\mathrm{RZ}$ modulation in Figure 3B under acceptable performance criteria [log $(\mathrm{BER}) \approx-6]$. This concludes that NRZ modulation outperforms RZ modulation by a notable increment of $4.5 \mathrm{~km}$ FSO link range. Henceforth, we have implemented the NRZ modulation format in the succeeding analysis. The clear eye diagrams at $14.5 \mathrm{~km}$ for both the spatial channels as shown in Figure 4 display a reliable transmission of $20 \mathrm{~Gb} / \mathrm{s}-20 \mathrm{GHz}$ information signal.

To determine how far and well the proposed link can function under four different dust storm situations, we draw the log of BER versus maximum reachable distance graphs. In addition, to demonstrate the reliability of our observations, we report the eye diagrams using the proposed system for each maximum reachable range with regard to specific dust conditions for both the channels.

Figure 5 shows a log of BER versus range curve under very light dust conditions, that is, $V \leq 10 \mathrm{~km}$. It is evident that the BER performance of the received signals deteriorated from a $\log$ (BER) of -21.69 at $2000 \mathrm{~m}$ to -9.24 at $2,500 \mathrm{~m}$ for the HG00 channel and -17.85 at $2000 \mathrm{~m}$ to -5.79 at $2,500 \mathrm{~m}$ for the HG01 channel, respectively. Figure 6 displays the eye diagram of the signals received from the HG00 and HG01 channels at a transmission range of 2,500 m under very light dust circumstances for the proposed design. The outcomes from Figures 5, 6 show $20 \mathrm{~Gb} / \mathrm{s}-20 \mathrm{GHz}$ information signal transmission around a distance of $2,500 \mathrm{~m}$ under very light dust conditions within standard performance criteria of log $(\mathrm{BER}) \approx-6$ and $\mathrm{Q}$ factor $\approx 6$.

The proposed NRZ-based MDM-RoFSO system performance in light dust conditions (i.e., $1 \mathrm{~km}<V<10 \mathrm{~km}$ ) as a function of the log (BER) and transmission range is presented in Figure 7. It can be found that the BER performance of the received signals declines from log (BER) of -23.51 at $750 \mathrm{~m}$ to -8.36 at $950 \mathrm{~m}$ for the HG00 channel and -20.27 at $750 \mathrm{~m}$ to -5.16 at $950 \mathrm{~m}$ for the HG01 channel, respectively. Figure 8 displays an eye diagram of the signals 

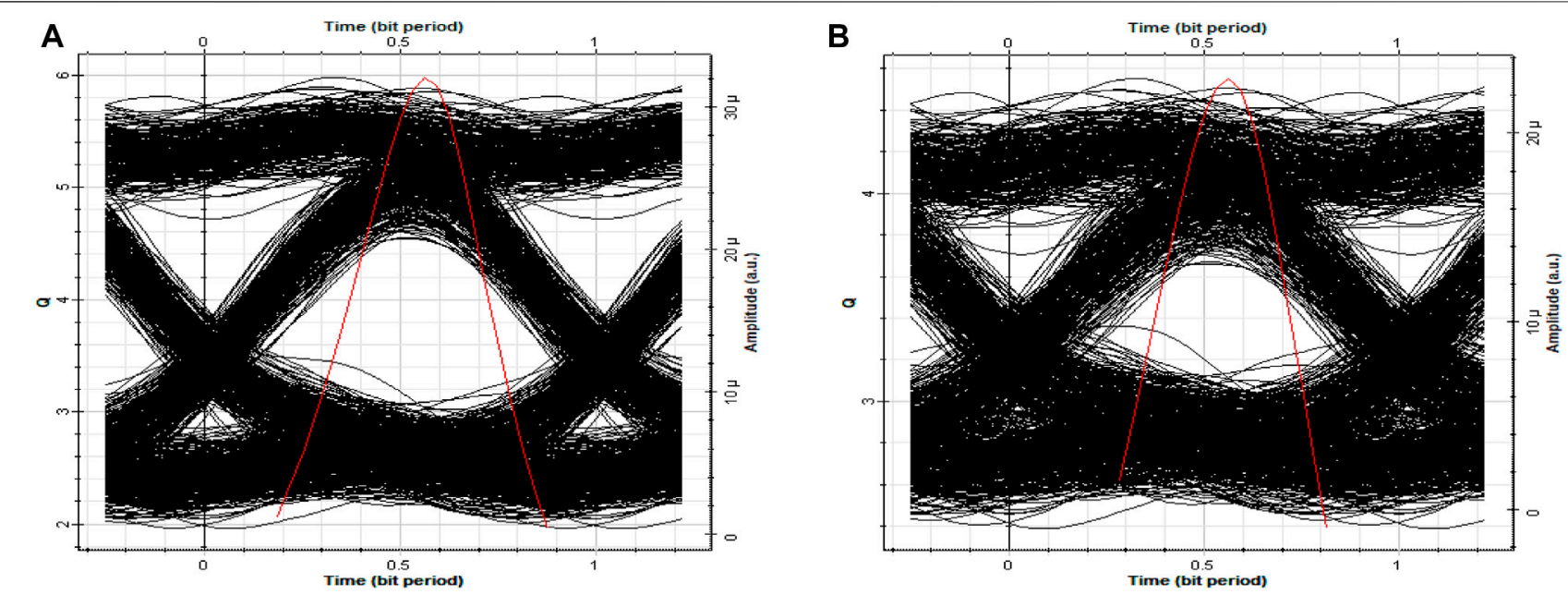

FIGURE 10 | Eye diagram using the proposed NRZ-based MDM-RoFSO system at 275 m transmission distance under moderate dust conditions for the (A) HG00 channel and (B) HG01 channel.

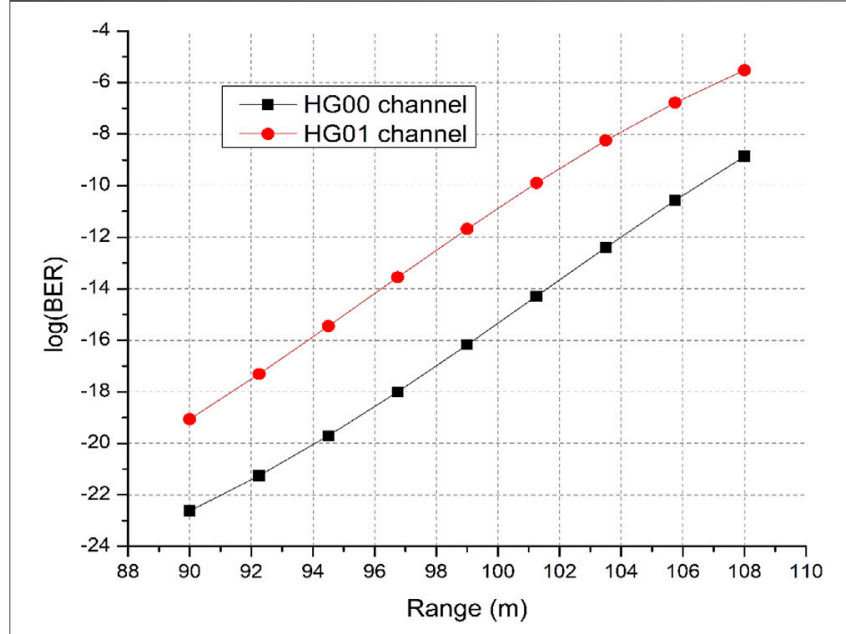

FIGURE $11 \mid \log (B E R)$ v/s transmission distance for dense dust.

received at $950 \mathrm{~m}$ for light dust conditions. The outcomes from Figures 7, 8 show $20 \mathrm{~Gb} / \mathrm{s}-20 \mathrm{GHz}$ information signal transmission around a distance of $950 \mathrm{~m}$ under very light dust conditions within standard performance criteria.
Figure 9 illustrates the log (BER) versus range graph under moderate dust conditions (i.e., $0.2 \mathrm{~km}<V<1 \mathrm{~km}$ ). As can be seen, the BER performance of the received signals reduces from a $\log$ (BER) of -23.36 at $225 \mathrm{~m}$ to -8.96 at $275 \mathrm{~m}$ for the HG00 channel and -20.05 at $225 \mathrm{~m}$ to -5.59 at $275 \mathrm{~m}$ for the HG01 channel, respectively. Figure 10 depicts the eye diagram of the two $10 \mathrm{~Gb} / \mathrm{s}-10 \mathrm{GHz}$ received signals from the HG00 and HG01 channels at a transmission distance of $275 \mathrm{~m}$ under moderate dust. The outcomes outlined in Figures 9, 10 reveal $20 \mathrm{~Gb} / \mathrm{s}$ $20 \mathrm{GHz}$ data transmission at $275 \mathrm{~m}$ in moderate dust situation with $\log (\mathrm{BER}) \approx-6$ and $\mathrm{Q}$ factor $\approx 6$.

The transmission performance of the proposed system under dense dust (i.e., $V<0.2 \mathrm{~km}$ ) as a function of the $\log (\mathrm{BER})$ and transmission range is shown in Figure 11. As can be noticed, the BER performance of the received signal deteriorates from a log (BER) of -22.62 at $90 \mathrm{~m}$ to -8.88 at $108 \mathrm{~m}$ for the HG00 channel and -19.06 at $90 \mathrm{~m}$ to -5.52 at $108 \mathrm{~m}$ for the HG01 channel, respectively. Figure 12 displays the eye diagram of the two signals transported over HG00 and HG01 channels at a distance of $108 \mathrm{~m}$ for a dense dust scenario. As follows from Figures 11, 12, $20 \mathrm{~Gb} /$ $\mathrm{s}-20 \mathrm{GHz}$ information is transported at $108 \mathrm{~m}$ under dense dust within acceptable performance limits using the proposed system. Nonetheless, there is no prominent increment in the maximum link reach under dense dust situations. Fortunately, the incident

TABLE 3 | Comparison of the transmission performance of the proposed system with recent works.

Parameter

Reference [7]

Transmission method

Data rate

Transmission power

Channel conditions and maximum range
OOK-based FSO link with MIMO and EDFA preamplifier $2.5 \mathrm{Gbps}$

$160 \mathrm{~mW}(22 \mathrm{dBm})$

Moderate dust- $2.3 \mathrm{~km}$ Heavy dust-380 m
Reference [23]

OOK-based FSO link with singlechannel transmission

$2.5 \mathrm{Gbps}$

$22 \mathrm{dBm}$

Light dust- $1 \mathrm{~km}$

Moderate dust- $800 \mathrm{~m}$

Heavy dust $<200$ m
Reference [33]

OOK-based WDM-FSO Link

1 Gbps

$50 \mathrm{dBm}$

Very light dust- $13.5 \mathrm{~km}$ Light dust-4.05 km

Moderate dust-1.05 km Heavy dust-0.405 km

\section{Proposed work}

OOK-based FSO Link employing MDM

20 Gbps

$14 \mathrm{dBm}$

Very light dust-2.5 km Light dust- $950 \mathrm{~m}$

Moderate dust-275 m

Heavy dust- $108 \mathrm{~m}$ 

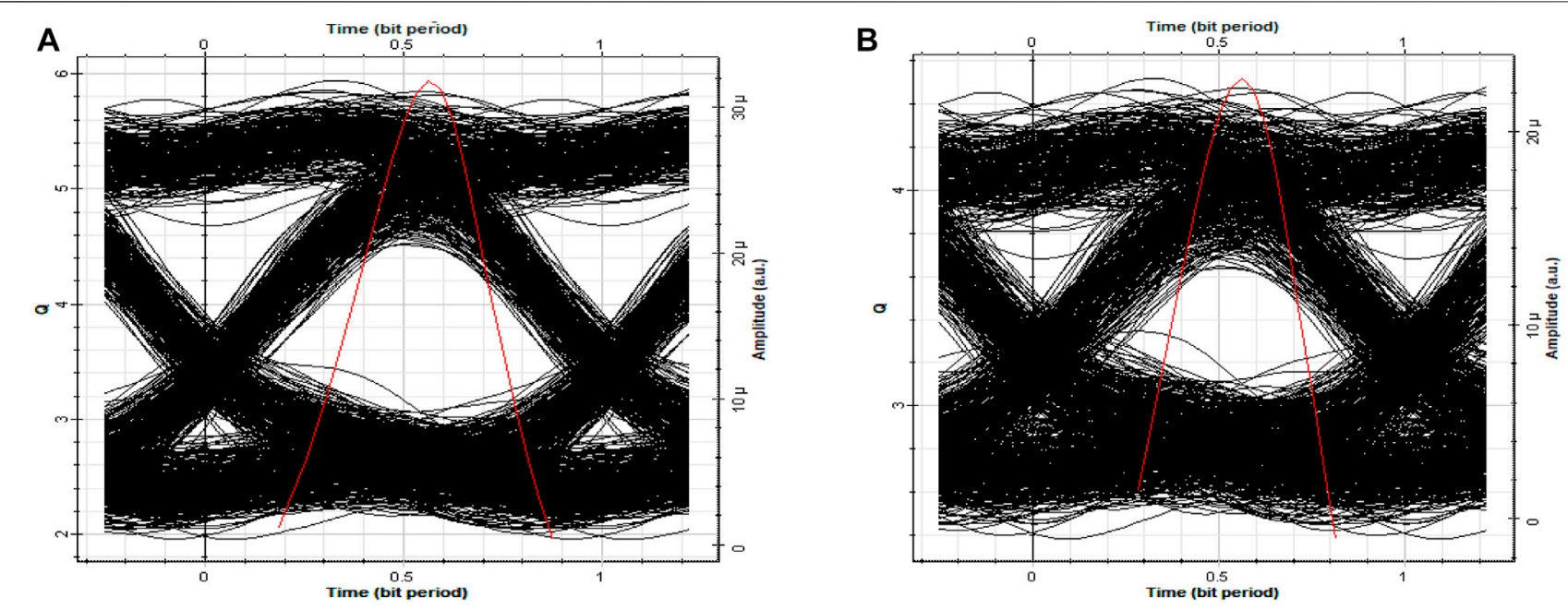

FIGURE 12 | Eye diagram using the proposed NRZ-based MDM-RoFSO link at $108 \mathrm{~m}$ transmission distance under dense dust for the (A) HG00 channel and (B) HG01 channel.

of dense dust waves is unusual and accounts for just $0.17 \%$ of the year time [36].

Table 3 compares the transmission performance of the proposed system with recent works and demonstrates that the proposed system achieves higher information capacity. The higher range in recent works can be attributed to the fact that the authors have considered higher transmission power, that is, $22 \mathrm{dBm}(\operatorname{Ref}[7,23])$ and $50 \mathrm{dBm}$ (Ref. [33]), as compared to $14 \mathrm{dBm}$ in this work.

\section{CONCLUSION}

RoFSO transmission systems integrated with MDM technology can provide a viable solution for providing secure biomedical sensor data transmission in medical facilities. In this work, an NRZ/RZ digital optical modulation schemes based MDM-RoFSO transmission system is proposed and investigated. The outcomes presented demonstrate that NRZ modulation performs significantly better in terms of providing extra $4.5 \mathrm{~km}$ coverage as compared to the RZ modulation format. We first investigated the performance reliability and availability of a NRZ-based MDMRoFSO transmission system in a dust environment. The results obtained show the likelihood of establishing short FSO networks under light and moderate dust circumstances. Nevertheless, rarely encountered dense dust scenarios can significantly affect the performance of MDM-RoFSO links. Additionally, because the $5 \mathrm{G}$ network uses ultra-compact

\section{REFERENCES}

1. Singh M, and Malhotra J. Performance Comparison of M-QAM and DQPSK Modulation Schemes in a $2 \times 20$ Gbit/s-40 GHz Hybrid MDM-OFDM-Based Radio over FSO Transmission System. Photon Netw Commun (2019) 38(3): 378-89. doi:10.1007/s11107-019-00861-z cells with modest separation, the proposed NRZ-based MDM-RoFSO link can prove to be a promising substitute to fiber cables in the fronthaul and backhaul sectors of nextgeneration wireless networks. In future works, the transmission capacity of the proposed system can further be enhanced by incorporating polarization multiplexing along with higher-order modulation schemes.

\section{DATA AVAILABILITY STATEMENT}

The original contributions presented in the study are included in the article/Supplementary Material, and further inquiries can be directed to the corresponding author.

\section{AUTHOR CONTRIBUTIONS}

MS: Conceptualization, methodology, investigation, writing-original draft, supervision, resources, project administration. SP: Methodology, investigation, writing-original draft, supervision. S: Methodology, investigation, data curation, visualization. SD: Investigation, data curation, writing-original draft. R: Investigation, data curation, visualization. AG: Writing-review and editing, validation, conceptualization. AM: Investigation, data curation, visualization. AM: Writing-review and editing, validation, conceptualization.

2. Singh M, and Malhotra J. Performance Comparison of High-Speed Long-Reach Mode Division Multiplexing-Based Radio over Free Space Optics Transmission System Using Different Modulation Formats under the Effect of Atmospheric Turbulence. Opt Eng (2019) 58(4):1. doi:10.1117/1.OE.58.4.046112

3. Sharma A, Malhotra J, Chaudhary S, and Thappa V. Analysis of $2 \times 10 \mathrm{Gbps}$ MDM Enabled Inter Satellite Optical Wireless Communication under the Impact of Pointing Errors. Optik (2021) 227:165250. doi:10.1016/j.ijleo.2020.165250 
4. Amphawan A, Chaudhary S, Ghassemlooy Z, and Neo T-K. $2 \times 2$-channel Mode-Wavelength Division Multiplexing in Ro-FSO System with PCF Mode Group Demultiplexers and Equalizers. Opt Commun (2020) 467:125539. doi:10.1016/j.optcom.2020.125539

5. Amphawan A, Chaudhary S, and Chan V. Optical Millimeter Wave Mode Division Multiplexing of LG and HG Modes for OFDM Ro-FSO System. Opt Commun (2019) 431:245-54. doi:10.1016/j.optcom.2018.07.054

6. Chaudhary S, and Amphawan A. Solid Core PCF-Based Mode Selector for MDM-Ro-FSO Transmission Systems. Photon Netw Commun (2018) 36: 263-71. doi:10.1007/s11107-018-0778-4

7. Alnajjar SH, Noori AA, and Moosa AA. Enhancement of FSO Communications Links under Complex Environment. Photonic Sens (2017) 7(2):113-22. doi:10.1007/s13320-017-0336-1

8. Algamal AA, Fayed HA, Mahmoud M, and Aly MH. Reliable FSO System Performance Matching Multi-Level Customer Needs in Alexandria City, Egypt, Climate: Sandstorm Impact with Pointing Error. Opt Quant Electron (2020) 52(18):1. doi:10.1007/s11082-020-02468-5

9. Esmail MA, Ragheb AM, Fathallah HA, Altamimi M, and Alshebeili SA. 5G-28 GHz Signal Transmission over Hybrid All-Optical FSO/RF Link in Dusty Weather Conditions. IEEE Access (2019) 7:24404-10. doi:10.1109/ ACCESS.2019.2900000

10. Baddock MC, Strong CL, Leys JF, Heidenreich SK, Tews EK, and Mctainsh GH. A Visibility and Total Suspended Dust Relationship. Atmos Environ (2014) 89:329-36. doi:10.1016/j.atmosenv.2014.02.038

11. Zhong H, Zhou J, Du Z, and Xie L. A Laboratory Experimental Study on Laser Attenuations by Dust/sand Storms. J Aerosol Sci (2018) 121:31-7. doi:10.1016/ j.jaerosci.2018.04.004

12. Ghassemlooy Z, Perez J, and Leitgeb E. On the Performance of FSO Communications Links under Sandstorm Conditions. In: Proceedings of the 12th International Conference on Telecommunications, 2013, June 26-28, Zagreb, Croatia. Zagreb (2013). p. 53-8.

13. Chaudhary S, Lin B, Tang X, Wei X, Zhou Z, Lin C, et al. 40 Gbps-80 GHz PSKMDM Based Ro-FSO Transmission System. Opt Quant Electron (2018) 50:321. doi:10.1007/s11082-018-1592-z

14. Chaudhary S, and Amphawan A. High-speed MDM-Ro-FSO System by Incorporating Spiral-Phased Hermite Gaussian Modes. Photon Netw Commun (2018) 35:374-80. doi:10.1007/s11107-017-0752-6

15. Chaudhary S, and Amphawan A. High-speed Millimeter Communication through Radio-Over-Free-Space-Optics Network by Mode-Division Multiplexing. Opt Eng (2017) 56(11):1. doi:10.1117/1.OE.56.11.116112

16. Chaudhary S, Tang X, and Wei X. Comparison of Laguerre-Gaussian and Donut Modes for MDM-WDM in OFDM-Ro-FSO Transmission System. AEU - Int J Elect Commun (2018) 93:208-14. doi:10.1016/j.aeue.2018.06.024

17. Kumar A, and Krishnan P. Performance Analysis of RoFSO Links with Spatial Diversity over Combined Channel Model for 5G in Smart City Applications. Opt Commun (2020) 466:125600. doi:10.1016/j.optcom.2020.125600

18. Bohren CF, and Huffman DR. Absorption and Scattering of Light by Small Particles. Germany: A Wiley-Interscience publication (1998). doi:10.1002/ 9783527618156

19. Kim II, Mcarthur B, Korevaar EJ, Street R, and Diego S. Comparison of Laser Beam Propagation at $785 \mathrm{Nm}$ and $1550 \mathrm{Nm}$ in Fog and Haze for Optical Wireless Communications. In: Proceeding SPIE 4214, Optical Communications III, 2001, February 6, Boston, MA, United States (2001). doi:10.1117/12.417512

20. Khalighi MA, Uysal M, Marseille C, and Engineering E. Survey on Free Space Optical Communication: A Communication Theory Perspective. IEEE Commun Surv Tutorials (2014) 16(4):2231-58. doi:10.1109/ COMST.2014.2329501

21. Hemmati H, Djordjevic IB, and Biswas A. Deep-Space Optical Communications: Future Perspectives and Applications. Proc IEEE (2011) 99(11):2020-39. doi:10.1109/JPROC.2011.2160609

22. Ijaz M, Ghassemlooy Z, Pesek J, Fiser O, Le Minh H, and Bentley E. Modeling of Fog and Smoke Attenuation in Free Space Optical Communications Link under Controlled Laboratory Conditions. J Lightwave Technol (2013) 31(11): 1720-6. doi:10.1109/JLT.2013.2257683
23. Esmail MA, Fathallah H, and Alouini M-S. Effect of Dust Storms on FSO Communications Links. In: 2016 4th International Conference on Control Engineering \& Information Technology (CEIT), 2016, December 16-18, Hammamet, Tunisia. Hammamet (2016). 1-6. doi:10.1109/ CEIT.2016.7929046

24. Pierce RM, Ramaprasad J, and Eisenberg EC. Optical Attenuation in Fog and Clouds. In: Proceeding SPIE, 4530, Optical Wireless Communications IV, 2001, November 27, Denver, CO, United States (2001). 58-71. doi:10.1117/ 12.449815

25. Prokes A. Atmospheric Effects on Availability of Free Space Optics Systems. Opt Eng (2009) 48(6):066001. doi:10.1117/1.3155431

26. Cao M, Wang H, Yao Y, and Hou S. Performance Evaluation of FSO Communications under Sand-Dust Conditions. Int J Antennas Propagation (2019) 2019:1-11. doi:10.1155/2019/2046896

27. Ricklin JC, Hammel SM, Eaton FD, and Lachinova SL. Atmospheric Channel Effects on Free-Space Laser Communication. J Optic Comm Rep (2006) 3: 111-58. doi:10.1007/s10297-005-0056-y

28. Esmail MA, Fathallah H, and Alouini M-S. An Experimental Study of FSO Link Performance in Desert Environment. IEEE Commun Lett (2016) 20(9): 1888-91. doi:10.1109/LCOMM.2016.2586043

29. Ghatak A, and Thyagarajan K. An Introduction to Fiber Optics. Cambridge: Cambridge University Press (1998). doi:10.1017/CBO9781139174770

30. Nyugen D-N, Bohata J, Spáčil J, Komanec M, Stevens N, Ghassemlooy Z, et al. Polarization Division Multiplexing-Based Hybrid Microwave Photonic Links for Simultaneous mmW and Sub 6-GHz Wireless Transmissions. IEEE Photon $J$ (2020) 12(6):1-16. doi:10.1109/JPHOT.2020.3036440

31. Kakati D, and Arya SC. A Full-Duplex Optical Fiber/wireless Coherent Communication System with Digital Signal Processing at the Receiver. Optik (2018) 171:190-9. doi:10.1016/j.ijleo.2018.05.140

32. Ghassemlooy Z, and Popoola WO. Terrestrial Free Space Optical Communications. In: S A Fares and F Adachi, editors. Mobile and Wireless Communication Network Layer and Circuit Level Design. London, United Kingdom: InTech (2010). doi:10.5772/7698

33. Ali MAA, Shaker FK, and Kadhum HA. Investigation and Analysis of Data Rate for Free Space Optical Communications System under Dust Conditions. Wirel Pers Commun (2020) 113:2327-38. doi:10.1007/s11277-020-07328-9

34. Mourka A, Mazilu M, Wright EM, and Dholakia K. Modal Characterization Using Principal Component Analysis: Application to Bessel, Higher-Order Gaussian Beams and Their Superposition. Sci Rep (2013) 3:1422. doi:10.1038/ srep01422

35. Amphawan A, and O'Brien D. Modal Decomposition of Output Field for Holographic Mode Field Generation in a Multimode Fiber Channel. In: International Conference on Photonics, 2010, July 5-7, Langkawi, Malaysia. Langkawi (2010). 1-5. doi:10.1109/ICP.2010.5604377

36. Alhaider MA. Radio Wave Propagation into Sandstorms-System Design Based on Ten-Years Visibility Data in Riyadh, Saudi Arabia. Int J Infrared Milli Waves (1986) 7(9):1339-59. doi:10.1007/BF01012054

Conflict of Interest: The authors declare that the research was conducted in the absence of any commercial or financial relationships that could be construed as a potential conflict of interest.

Publisher's Note: All claims expressed in this article are solely those of the authors and do not necessarily represent those of their affiliated organizations, or those of the publisher, the editors, and the reviewers. Any product that may be evaluated in this article, or claim that may be made by its manufacturer, is not guaranteed or endorsed by the publisher.

Copyright $\odot 2021$ Singh, Pottoo, Suvidhi, Dewra, Rishabh, Grover, Manikandan and Sheetal. This is an open-access article distributed under the terms of the Creative Commons Attribution License (CC BY). The use, distribution or reproduction in other forums is permitted, provided the original author(s) and the copyright owner(s) are credited and that the original publication in this journal is cited, in accordance with accepted academic practice. No use, distribution or reproduction is permitted which does not comply with these terms. 